\title{
Numerical study of the coupled time-dependent Gross-Pitaevskii equation: Application to Bose-Einstein condensation
}

\author{
Sadhan K. Adhikari \\ Instituto de Física Teórica, Universidade Estadual Paulista, 01.405-900 São Paulo, São Paulo, Brazil
}

(Received 20 June 2000; published 20 April 2001)

\begin{abstract}
We present a numerical study of the coupled time-dependent Gross-Pitaevskii equation, which describes the Bose-Einstein condensate of several types of trapped bosons at ultralow temperature with both attractive and repulsive interatomic interactions. The same approach is used to study both stationary and time-evolution problems. We consider up to four types of atoms in the study of stationary problems. We consider the time-evolution problems where the frequencies of the traps or the atomic scattering lengths are suddenly changed in a stable preformed condensate. We also study the effect of periodically varying these frequencies or scattering lengths on a preformed condensate. These changes introduce oscillations in the condensate, which are studied in detail. Good convergence is obtained in all cases studied.
\end{abstract}

DOI: 10.1103/PhysRevE.63.056704

PACS number(s): 02.70.-c, 02.60.Lj, 03.75.Fi

\section{INTRODUCTION}

The experimental detection [1] of Bose-Einstein condensation (BEC) at ultralow temperature in dilute bosonic atoms (alkali and hydrogen atoms) employing magnetic traps have spurred intense theoretical activities on various aspects of the condensate [2-8]. Many properties of the condensate are usually described by the nonlinear mean-field GrossPitaevskii (GP) equation [7,9]. The GP equation in both time-dependent and independent forms is formally similar to the Schrödinger equation including a nonlinear term $[3,4,6]$.

More recently, there has been experimental realization of BEC involving two types of atoms [10-12]. In the actual experiment ${ }^{87} \mathrm{Rb}$ atoms formed in the $F=1, m=-1$, and $F=2, \quad m=1$ states by the use of a laser, served as two different species of atoms, where $F$ and $m$ are the total angular momentum and its projection [11]. In another experiment a coupled BEC was formed with the ${ }^{87} \mathrm{Rb}$ atoms in the $F=1, m=-1$, and $F=2, \quad m=2$ states [7,12]. Experimentally, it is possible to use the same magnetic trap to confine the atoms in two quantum states, which makes this study easier technically compared to the formation of a BEC with two different types of atoms requiring two different trapping mechanisms. It has also been found in these studies $[11,12]$ that the ${ }^{87} \mathrm{Rb}$ atoms have a repulsive interaction in all three states considered above. These experiments initiated theoretical activities in multicomponent BEC described by the coupled GP equation [13].

A numerical study of the time-dependent coupled GP equation is interesting as this can provide solution to many time-evolution problems involving more than one type of atoms forming a BEC. The solution of the coupled nonlinear GP equation is nontrivial [14] and here we undertake the challenging task of the numerical study of these timeevolution problems [5].

In a multicomponent BEC, the main feature is the coupling between different types of condensates, which can lead to new effects associated with this surely much richer situation nonexistent in a single-component BEC. We list some interesting possibilities below, which we shall investigate numerically in this paper. It is possible to have a distinct trapping frequency for each of the components, one of which can be suddenly altered experimentally [16]. Of course, this change would affect the component of the BEC directly trapped by this field. However, it is more interesting to study how this sudden change affects the other component of BEC not directly trapped by this field. Also it is possible to suddenly vary [17] the atomic scattering length of one of the species and study its effect on the other component. The above variation of one of the trap frequencies or scattering lengths can be carried through in a periodic fashion and its effect on the other component can be studied. The studies mentioned above are peculiar to a coupled BEC and are of interest as it is now possible to vary both the trap frequencies [16] as well as the scattering lengths $[7,11,17]$ both abruptly or in a periodic fashion. These effects do not have any analogue in the uncoupled BEC and we motivate the present investigation with special emphasis on these effects in this paper. We investigate these time-evolution problems using a set of two coupled GP equations in the purely repulsive case. In addition we study the stationary solution to the coupled GP equation describing a multicomponent condensate, where we consider up to four components.

We solve the coupled BEC problem using the timedependent coupled GP equation in cases of attractive and repulsive atomic interactions by discretization with the Crank-Nicholson-type rule complimented by the known boundary conditions at origin and infinity [15]. This procedure leads to good convergence for both the stationary and time-evolution problems.

First we consider stationary coupled condensates under the action of trap potentials. Stable and converged numerical results are obtained for up to four coupled equations in the repulsive case and two in the attractive case. The timedependent GP equation is directly solved to obtain the full time-dependent solution in the case of stationary problems, from which a trivial time-dependent phase factor is separated and the stationary solution obtained as in the uncoupled case [5].

We also study the numerical stability of the calculational scheme, which is more difficult to obtain when the nonlin- 
earity is large. For this purpose we only consider repulsive interatomic interactions in the time-evolution problems where condensates with large nonlinearity can be formed. In the case of attractive interaction a large nonlinearity leads to the collapse of the condensate [8].

In Sec. II we present the coupled time-dependent GP equation that we use. In Sec. III we describe the numerical method in some detail. In Sec. IV we report the numerical results for the stationary case and in Sec. V we report a study of three types of time-evolution problems. Finally, in Sec. V we give a summary of our investigation.

\section{NONLINEAR COUPLED GROSS-PITAEVSKII EQUATION}

The GP equation [9] for a coupled trapped Bose-Einstein condensate at zero temperature is written as [13]

$$
\begin{gathered}
{\left[-\frac{\hbar^{2}}{2 m} \nabla^{2}+\frac{1}{2} c_{i} m \omega^{2} r^{2}+\sum_{l=1}^{M} g_{i l} N_{l}\left|\Psi_{l}(\mathbf{r}, \tau)\right|^{2}\right.} \\
\left.-i \hbar \frac{\partial}{\partial \tau}\right] \Psi_{i}(\mathbf{r}, \tau)=0, \ldots, M,
\end{gathered}
$$

where $\Psi_{i}(\mathbf{r}, \tau)$ at position $\mathbf{r}$ and time $\tau$ is the wave function for the component $i$ of the condensate, $m$ is the mass of a single bosonic atom, $N_{l}$ the number of condensed atoms of type $l, \quad M$ the number of types of atoms, $c_{i} m \omega^{2} r^{2} / 2$ the attractive harmonic-oscillator trap potential, and $\omega$ the oscillator frequency. The parameter $c_{i}$ has been introduced to independently modify the frequency of the harmonicoscillator trap for each type of atoms. Here $g_{i l}$ $=4 \pi \hbar^{2} a_{i l} / m$ is the coupling constant for elastic interaction between atoms of types $i$ and $l$, where $a_{i l}$ is the corresponding scattering length. The masses of different types of atoms are taken to be equal, as this is necessary while considering the coupled BEC formed of different spin states of ${ }^{87} \mathrm{Rb}$ - one of the most important realizations to date. In this paper we shall not allow the transition of one type of atoms of the BEC to the other and take the number of atoms of each component to be constant as in the experiment of Ref. [12].

Here we shall be interested in the spherically symmetric solution $\Psi_{i}(\mathbf{r}, \tau) \equiv \psi_{i}(r, \tau)$ to Eq. (2.1), which can be written as

$$
\begin{gathered}
{\left[-\frac{\hbar^{2}}{2 m} \frac{1}{r} \frac{\partial^{2}}{\partial r^{2}} r+\frac{1}{2} c_{i} m \omega^{2} r^{2}+\sum_{l=1}^{M} g_{i l} N_{l}\left|\psi_{l}(r, \tau)\right|^{2}\right.} \\
\left.-i \hbar \frac{\partial}{\partial \tau}\right] \psi_{i}(r, \tau)=0, \ldots, M .
\end{gathered}
$$

The above limitation to the spherically symmetric solution (in the zero angular momentum state) reduces the coupled GP equation to a one-dimensional coupled partial differential equation.

As in Refs. [4,5] it is convenient to use dimensionless variables defined by $x=\sqrt{2} r / a_{\mathrm{ho}}$, and $t=\tau \omega$, where $a_{\mathrm{ho}}$ $\equiv \sqrt{\hbar /(m \omega)}$, and $\phi_{i}(x, t)=x \psi_{i}(r, \tau)\left(\sqrt{2} \pi a_{\mathrm{ho}}^{3}\right)^{1 / 2}$. In terms of these variables Eq. (2.2) becomes

$$
\left[-\frac{\partial^{2}}{\partial x^{2}}+\frac{1}{4} c_{i} x^{2}+\sum_{l=1}^{M} n_{i l} \frac{\left|\phi_{l}(x, t)\right|^{2}}{x^{2}}-i \frac{\partial}{\partial t}\right] \phi_{i}(x, t)=0,
$$

where $n_{i l} \equiv 2 \sqrt{2} N_{l} a_{i l} / a_{\text {ho }}$ is the reduced number of particles and this number could be negative (positive) when the corresponding scattering length is negative (positive), representing an attractive (repulsive) interatomic interaction. The normalization of the wave function is $\int_{0}^{\infty}\left|\phi_{i}(x, t)\right|^{2} d x=1$ and its root-mean-square (rms) radius $x_{\mathrm{rms}}^{(i)}$ is given by

$$
x_{\mathrm{rms}}^{(i)}=\left[\int_{0}^{\infty} x^{2}\left|\phi_{i}(x, t)\right|^{2} d x\right]^{1 / 2} .
$$

\section{NUMERICAL METHOD}

To solve Eq. (2.3) numerically one needs the proper boundary conditions at $x \rightarrow 0$ and $\infty$. For a confined condensate, for a sufficiently large $x, \quad \phi_{i}(x, t)$ must vanish asymptotically. Hence the nonlinear term proportional to $\left|\phi_{i}(x, t)\right|^{3}$ can eventually be neglected in the GP equation for large $x$. Consequently the asymptotic form of the physically acceptable solution is given by $\lim _{x \rightarrow \infty}\left|\phi_{i}(x, t)\right| \sim \exp \left(-x^{2} / 4\right)$. Next we consider Eq. (2.3) as $x \rightarrow 0$. The nonlinear term approaches a constant in this limit because of the regularity of the wave function at $x=0$. Then one has the condition $\left|\phi_{i}(0, t)\right|=0$.

A convenient way to solve Eq. (2.3) numerically is to discretize it in both space and time and reduce it to a set of algebraic equations, which could then be solved by using the known asymptotic boundary conditions. The procedure is similar to that in the uncoupled case [5]. We discretize Eq. (2.3) by using a space step $h$ and time step $\Delta$ with a finite difference scheme using the unknown $\left(\phi_{i}\right)_{p}^{k}$, which will be approximation of the exact solution $\phi_{i}\left(x_{p}, t_{k}\right)$, where $x_{p}$ $=p h$ and $t_{k}=k \Delta$. The time derivative in Eq. (2.3) involves the wave function at times $t_{k}$ and $t_{k+1}$. As in the uncoupled case we express the wave functions and their derivatives in Eq. (2.3) in terms of the average over times $t_{k}$ and $t_{k+1}$ [15] and the resultant scheme leads to accurate results and good convergence. In practice we use the following CrankNicholson-type scheme [15] to discretize the partial differential equation (2.3)

$$
\begin{aligned}
i \frac{\left(\phi_{i}\right)_{p}^{k+1}-\left(\phi_{i}\right)_{p}^{k}}{\Delta}= & -\frac{1}{2 h^{2}}\left[\left(\phi_{i}\right)_{p+1}^{k+1}-2\left(\phi_{i}\right)_{p}^{k+1}+\left(\phi_{i}\right)_{p-1}^{k+1}\right. \\
& \left.+\left(\phi_{i}\right)_{p+1}^{k}-2\left(\phi_{i}\right)_{p}^{k}+\left(\phi_{i}\right)_{p-1}^{k}\right] \\
& +\frac{1}{2}\left[\frac{c_{i} x_{p}^{2}}{4}+\sum_{l=1}^{M} n_{i l} \frac{\left|\left(\phi_{l}\right)_{p}^{k}\right|^{2}}{x_{p}^{2}}\right] \\
& \times\left[\left(\phi_{i}\right)_{p}^{k+1}+\left(\phi_{i}\right)_{p}^{k}\right]
\end{aligned}
$$


Considering that the wave-function components $\phi_{i}$ are known at time $t_{k}$, Eq. (3.1) is an equation in the unknowns- $\left(\phi_{i}\right)_{p+1}^{k+1},\left(\phi_{i}\right)_{p}^{k+1}$, and $\left(\phi_{i}\right)_{p-1}^{k+1}$. In a lattice of $N$ points, Eq. (3.1) represents a tridiagonal set for $p$ $=2,3, \ldots,(N-1)$ for a specific component $\phi_{i}$. This set has a unique solution if the wave functions at the two end points $\left(\phi_{i}\right)_{1}^{k+1}$ and $\left(\phi_{i}\right)_{N}^{k+1}$ are known [15]. In the present problem these values at the end points are provided by the known asymptotic conditions.

To find the ground state of the condensate we start with the analytically known properly normalized wave functions of the uncoupled harmonic-oscillator problems described by Eq. (2.3) with $n_{i l}=0$. We then repeatedly propagate these solutions in time using the Crank-Nicholson-type algorithm (3.1). Starting with $n_{i l}=0$, at each time step we increase or decrease the nonlinear parameter $n_{i l}$ by an amount $\Delta_{1}$. This procedure is continued until the desired final value of $n_{i l}$ is reached. The resulting solution is the ground state of the condensate corresponding to the specific nonlinearity.

The time-dependent approach is the most suitable for solving time-evolution problems. In the present paper we consider only evolution problems starting from a stable condensate at $t=0$. In these cases the stationary problem is solved first and the wave function so obtained serves as the starting wave function for the time-evolution problem.

\section{RESULTS FOR THE STATIONARY PROBLEM}

First we consider the stationary ground-state solution of Eq. (2.3) in cases of both attractive and repulsive interactions using two and four coupled equations. The numerical integration was performed up to $x_{\max }=15$ with $h=0.0001$ using time step $\Delta=0.05$ and the parameter $\Delta_{1}=0.01$. After some experimentation we find that good convergence is obtained with parameters $\Delta$ and $\Delta_{1}$ near these values. The convergence is fast for small nonlinearity. The final convergence of the scheme breaks down if nonlinearity is too large. In practice these difficulties start for $n_{i i}>20$ for the ground state for a repulsive interaction in a computational analysis in double precision. For an attractive interaction the coupled GP equation does not sustain a large nonlinearity $\left|n_{i l}\right|$ and leads to collapse. Except for values of nonlinearity near collapse, the GP equation in the attractive case leads to good convergence.

\section{A. Repulsive atomic interaction}

In most of the experimental realization of BEC in trapped atoms, the interatomic interaction is repulsive and we consider this case first. We consider the simple case of two coupled GP equations in the case of repulsive interaction with (a) $n_{11}=n_{22}=10, n_{12}=n_{21}=5, c_{1}=1$, and $c_{2}=0.25$, (b) $n_{11}=n_{22}=5, n_{12}=n_{21}=100, c_{1}=1$, and $c_{2}=0.25$, and (c) $n_{11}=n_{22}=10, n_{12}=n_{21}=5$, and $c_{1}=c_{2}=1$. In this case all interactions are repulsive corresponding to the positive sign of all $n_{i l} \equiv 2 \sqrt{2} N_{i} a_{i l} / a_{\text {ho }}$. Although these parameters are in dimensionless units, it is easy to associate them to an actual physical problem of experimental interest. For example, for the mixture of $|F=1, m=-1\rangle$ and $|F=2, m=1\rangle{ }^{87} \mathrm{Rb}$ states, the ratio of scattering lengths

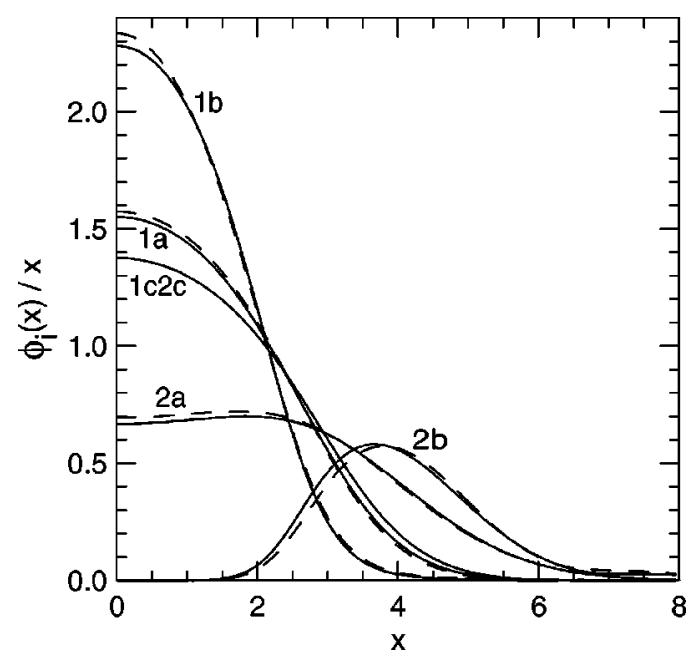

FIG. 1. Wave-function components $\phi_{1}$ (label 1) and $\phi_{2}$ (label 2) for two coupled GP equations with (a) $n_{11}=n_{22}=10, \quad n_{12}$ $=n_{21}=5, \quad c_{1}=1, \quad c_{2}=0.25 ;$ (b) $n_{11}=n_{22}=5, \quad n_{12}=n_{21}=100$, $c_{1}=1, c_{2}=0.25$; and (c) $n_{11}=n_{22}=10, n_{12}=n_{21}=5, c_{1}=1$, and $c_{2}=1$ calculated with $\Delta=0.05$ and $\Delta_{1}=0.01$ (full line); $\Delta=0.05$ and $\Delta_{1}=0.015$ (dashed line). In case (c) only the results for $\Delta_{1}$ $=0.01$ are shown.

$a_{|1,-1\rangle} / a_{|2,1\rangle}=1.062$ [11]. If we label the $|1,-1\rangle$ state by 1 and the $|2,1\rangle$ state by 2 , and consider $a_{11} / a_{\mathrm{ho}} \simeq a_{22} / a_{\mathrm{ho}}$ $\simeq 0.002$, then $n_{11}=n_{22}=10$ corresponds to $N_{1} \simeq N_{2} \simeq 1770$. This estimate gives an idea of the actual experimental condition that the present set of parameters simulate. The three models considered above can simulate actual experimental situations composed of two states of ${ }^{87} \mathrm{Rb}$. The different values of $n_{12}$ and $n_{21}$ considered above correspond to different possible unknown repulsive interactions among the two species of condensates. It is realized from the coupled GP equation that in case of model (c), $\phi_{1}=\phi_{2}$. We show results for the two components of the wave function for two sets of values of $\Delta_{1}: 0.01$ (full line) and 0.015 (dashed line) in Fig. 1. The difference between the two sets of results increases as the nonlinearity of the GP equation given by $n_{i l}$ increases, e.g., for the case (b) above compared to (a). The difference reduces to zero as the nonlinearity decreases.

Next we consider the more complicated case of four coupled GP equations with repulsive atomic interaction. This is a purely theoretical case with no experimental analogue, as all experiments to date are limited to two coupled condensates only. In this case the numerical method works in the same fashion and good convergence is obtained with moderate values of nonlinearity. Again we consider the case of repulsive interaction between all possible pairs. In this case, in the four-component model, we take $n_{11}=4, n_{22}=5, n_{33}$ $=6, n_{44}=8$, and $n_{i l}=2, i \neq l ; c_{1}=4, c_{2}=1, c_{3}=4$, and $c_{4}$ $=0.25$. The solution for the wave-function components obtained with $\Delta=0.05$ and $\Delta_{1}=0.01$ (full line), and $\Delta_{1}$ $=0.015$ (dashed line), are shown in Fig. 2. The maximum difference between the two calculations is about $6 \%$ for the largest component $\left(\phi_{1}\right)$ near $x=0$, although the average difference is much smaller, as can be seen in Fig. 2. 


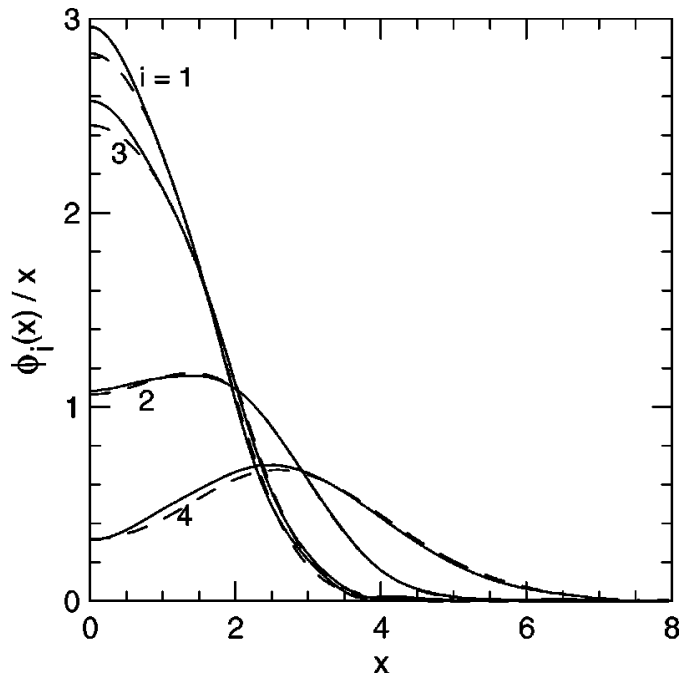

FIG. 2. Wave-function components $\phi_{1}$ (label 1), $\phi_{2}$ (label 2), $\phi_{3}$ (label 3), and $\phi_{4}$ (label 4) for four coupled GP equations with $n_{11}=4, n_{22}=5, n_{33}=6, n_{44}=8$, and $n_{i l}=2, i \neq l ; c_{1}=4, c_{2}=1$, $c_{3}=4$, and $c_{4}=0.25$ calculated with $\Delta=0.05$ and $\Delta_{1}=0.01$ (full line); $\Delta=0.05$ and $\Delta_{1}=0.015$ (dashed line).

\section{B. Attractive atomic interaction}

The case of attractive atomic interaction demands special attention as one can have the phenomenon of collapse in this case. We consider the case of two coupled GP equations with attractive interactions between like atoms $i i, \quad i=1,2$, and with repulsive interactions between unlike atoms $i j, \quad i \neq j$. In this case some of the atomic interactions are attractive in nature. Consequently, with large (attractive) nonlinearity, the system may undergo collapse and for stable stationary solution of the GP equation, the nonlinearity should be maintained small. We consider the following three cases: (a) $n_{11}$ $=-1, n_{22}=-1.5, n_{12}=n_{21}=4, c_{1}=4$, and $c_{2}=1$; (b) $n_{11}$ $=-1, n_{22}=-1.5, n_{12}=n_{21}=80, c_{1}=4$, and $c_{2}=1$; (c) $n_{11}=-1, n_{22}=-1.5, n_{12}=n_{21}=100, c_{1}=4$, and $c_{2}=1$. The only observed case of BEC with attractive interaction is the case of ${ }^{7} \mathrm{Li}$ with $a / a_{\mathrm{ho}} \simeq-0.0005$ [7]. If we label this state by 1 then $n_{11}=-1$ corresponds to the actual particle number $N_{1}=700$. In an uncoupled condensate of ${ }^{7} \mathrm{Li}$ the BEC collapses for more than 1400 atoms. Although there has been no experimental realization of coupled BEC in the case of attractive interaction, the parameters cited above may simulate the BEC of ground-state atoms of ${ }^{7} \mathrm{Li}$ coupled to one of its excited states, where the atomic interaction is also attractive. If we assume that the excited-state atoms have the same value of $a / a_{\text {ho }}$ as in the ground state then $n_{22}=-1.5$ corresponds to the number of atoms $N_{2}=1500$ in the excited state where the excited state is labeled by the index 2 .

The wave-function components in this case are shown in Fig. 3. As for a stable stationary solution the nonlinearity in this case has to be smaller than in the purely repulsive case, numerically it is easier to obtain precise solution except for values of the nonlinearity close to (and beyond) collapse. The parameters above in cases (a), (b), and (c) are chosen to illustrate the collapse of the system arising from the divergence of the first component of the wave function $\left(\phi_{1}\right)$. The

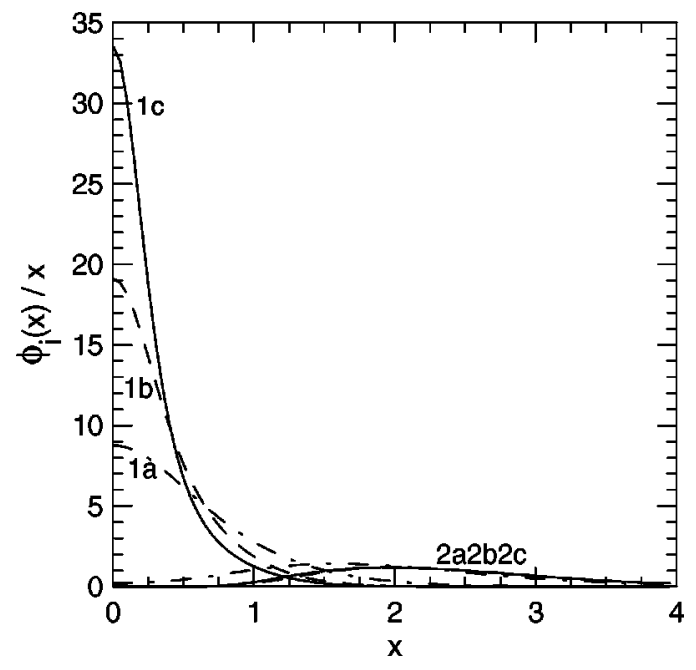

FIG. 3. Wave-function components $\phi_{1}$ (label 1) and $\phi_{2}$ (label 2) for two coupled GP equations with (a) $n_{11}=-1, n_{22}=-1.5$, $n_{12}=n_{21}=4, c_{1}=4$, and $c_{2}=1$ (dashed-dotted line); (b) $n_{11}=$ $-1, n_{22}=-1.5, n_{12}=n_{21}=80, c_{1}=4$, and $c_{2}=1$ (dashed line); (c) $n_{11}=-1, n_{22}=-1.5, n_{12}=n_{21}=100, c_{1}=4$, and $c_{2}=1$ (full line) calculated with $\Delta=0.05$ and $\Delta_{1}=0.01$.

nonlinear couplings $n_{12}$ and $n_{21}$ are increased as we move from case (a) to (b) and then to (c). Other parameters of the model are kept fixed. Because of the attractive interactions in this case, the system moves towards collapse as we move from case (a) to (c) through (b). The central density corresponding to $\phi_{1}$ increases (eventually tends to infinity) and the rms radius of the system decreases (eventually tends to zero) with the increase of nonlinearity. This is clear from a comparison of Fig. 3 with Figs. 1 and 2. The very large central value of the wave function $\phi_{1}(\sim 35)$ and its small radial extension, indicates a large central density and a small rms radius.

Finally, we consider the case of two interacting systems with all interactions repulsive. In this case the system is more vulnerable to collapse if the nonlinearity is large. We consider the following three sets of parameters in this case for which we show the solution in Fig. 4: (a) $n_{11}=-1, n_{22}=$ $-1, n_{12}=n_{21}=-0.4, c_{1}=4$, and $c_{2}=0.25$; (b) $n_{11}=-1$, $n_{22}=-1, n_{12}=n_{21}=-0.5, c_{1}=4$, and $c_{2}=0.25$; (c) $n_{11}=$ $-1, n_{22}=-1, n_{12}=n_{21}=-0.55, c_{1}=4$, and $c_{2}=0.25$. These parameters simulate the possible coupled BEC composed of the attractive ground and excited states of ${ }^{7} \mathrm{Li}$, where the interaction between a ground- and an excited-state atom is also taken to be attractive. It is possible to calculate the number of the two types of atoms as in the discussion related to Fig. 3. Here the nonlinearity increases as we move from case (a) to (b) and then to (c), and consequently, the wave-function components become more and more localized with a large central density and small rms radii signaling the onset of collapse of the system. This is clear from Fig. 4. In case (c) the nonlinearity is the highest and one is closer to collapse. However, there is a difference between the two collapses shown in Figs. 3 and 4. In Fig. 3 the route to collapse is manifested through a singular behavior of component $\phi_{1}$ of the wave function; in Fig. 4, both components 


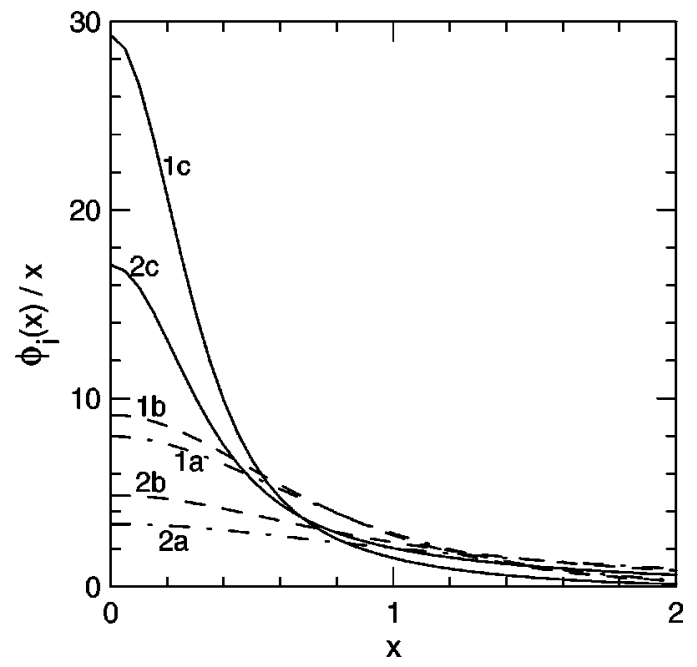

FIG. 4. Wave-function components $\phi_{1}$ (label 1) and $\phi_{2}$ (label 2) for two coupled GP equations with (a) $n_{11}=-1, n_{22}=-1, n_{12}$ $=n_{21}=-0.4, c_{1}=4$, and $c_{2}=0.25$ (dashed-dotted line); (b) $n_{11}=$ $-1, n_{22}=-1, n_{12}=n_{21}=-0.5, c_{1}=4$, and $c_{2}=0.25$ (dashed line); (c) $n_{11}=-1, n_{22}=-1, n_{12}=n_{21}=-0.55, c_{1}=4$, and $c_{2}$ $=0.25$ (full line) calculated with $\Delta=0.05$ and $\Delta_{1}=0.01$.

$\phi_{1}$ and $\phi_{2}$ exhibit the singular behavior. The spacial extension of the wave-function components close to collapse in Figs. 3 and 4 is much smaller than the wave-function components in the purely repulsive cases shown in Figs. 1 and 2.

\section{Estimate of numerical error}

It is appropriate to comment quantitatively on the numerical accuracy of the present method. If we iterate the final solution in time without changing the nonlinearity, the numerical result keeps on oscillating with a small amplitude around the converged value. This oscillation gives a good estimate of the numerical error of the method. This error manifests in a different way in Fig. 1, where we have varied $\Delta_{1}$. The numerical solution of the time-dependent method is independent of the space step $h$ provided that a typical value around $h=0.0001$ is employed as in the present paper. No visible difference in the solution is found if $h$ is increased by a factor of 2 . However, the above oscillations with time iteration are sensitive to the parameters $\Delta$ and $\Delta_{1}$. The values of these parameters $\left(\Delta=0.05\right.$ and $\left.\Delta_{1}=0.01\right)$ are chosen to minimize the oscillation of the results with time iterations. The oscillation increases if larger or smaller values of one or both of these parameters are employed and can really be large if an improper value of step $\Delta$ or $\Delta_{1}$ is chosen. This oscillation is quite similar to that in the uncoupled case studied in detail in Ref. [5].

From Figs. 1 and 2 we find that the error in the wavefunction component $\left|\phi_{i}(x, t) / x\right|$ as a function of $x$ is the largest at $x=0$. In Table I we show the percentage error of $\lim _{x \rightarrow 0}\left|\phi_{i}(x, 0) / x\right|$, defined by $\mathcal{E} \equiv 100\left[\left|\phi_{i}(0, t)\right|\right.$ $\left.-\left|\phi_{i}(0,0)\right|\right] /\left|\phi_{i}(0,0)\right|$ at those iterations where this error is maximum. For illustration we consider the models (a) discussed in Figs. 1 and 4. At positive $\mathcal{E}$ there is overshooting and at negative $\mathcal{E}$ there is undershooting. Between a positive $\mathcal{E}$ and a negative $\mathcal{E}$ there is a zero of $\mathcal{E}$ denoting zero error. We find that the wave functions oscillate with time around the stationary solution. The maximum reported error is about $6 \%$. Considering that we are dealing with coupled nonlinear equations these errors are well within the acceptable limit. The errors shown in Table I would also be the typical errors in time-evolution problems with the same nonlinearity that we study in Sec. V. From Table I we see that the period of oscillation of the result varies from one case to another. However, the error increases as the nonlinearity increases or as the system approaches collapse in the case of attractive interaction. For example, in models (c) of Figs. 3 and 4, which are close to collapse corresponding to almost maximum permissible nonlinearity, the error increases quickly with time iteration and a large numerical error could be generated easily.

\section{RESULTS FOR THE TIME-EVOLUTION PROBLEM}

Now we consider three types of time-evolution problems, some of which could possibly be studied experimentally. As the repulsive case leads to more stable configuration of the condensate, in this paper we consider only this case in the process of time evolution. The attractive case of coupled BEC is also very interesting from a physical point of view because of the occurrence of collapse. We have performed a study of the dynamics of collapse in coupled BEC using the present numerical method, which will be reported elsewhere. The two types of parameters that can be varied in the timeevolution study are the frequencies of the harmonicoscillator traps and the different scattering lengths. Recently, it has been possible to vary the scattering length experimentally by varying an external field $[11,12]$. It is also possible to vary the trap frequency by varying the currents in the

TABLE I. Percentage error $\mathcal{E} \equiv 100\left[\left|\phi_{i}(0, T)\right|-\left|\phi_{i}(0,0)\right|\right] /\left|\phi_{i}(0,0)\right|$ of $\left|\phi_{i}(0, T)\right|(i=1,2)$ at successive reduced times $T \equiv 0.05$, where this error is maximum, calculated with $\Delta=0.05$ and $\Delta_{1}=0.01$. The cases considered correspond to model (a) of Figs. 1 and 4 .

\begin{tabular}{|c|c|c|c|c|c|c|c|c|c|c|c|c|c|c|c|c|c|c|c|}
\hline Fig. 1 & $T$ & 0 & 19 & 42 & 67 & 101 & 125 & 165 & 190 & 225 & 249 & 271 & 311 & 337 & 371 & 395 & 414 & 458 & 483 \\
\hline$\phi_{1}(\mathrm{a})$ & $\mathcal{E}$ & 0 & -1.7 & 2.5 & -1.0 & 3.5 & -1.4 & 3.2 & -1.9 & 2.9 & -2.7 & 2.1 & -3.2 & 4.0 & -2.1 & 4.2 & -1.8 & 5.4 & -3.7 \\
\hline Fig. 1 & $T$ & 0 & 36 & 85 & 163 & 219 & 275 & 353 & 395 & 477 & & & & & & & & & \\
\hline$\phi_{2}(\mathrm{a})$ & $\mathcal{E}$ & 0 & 5.4 & -4.9 & 6.2 & -4.2 & 5.5 & -4.2 & 6.2 & -4.6 & & & & & & & & & \\
\hline Fig. 4 & $T$ & 0 & 13 & 110 & 192 & 250 & 318 & 376 & 467 & & & & & & & & & & \\
\hline$\phi_{1}(\mathrm{a})$ & $\mathcal{E}$ & 0 & 1.2 & -0.6 & 1.0 & -0.5 & 1.0 & -0.3 & 0.9 & & & & & & & & & & \\
\hline Fig. 4 & $T$ & 0 & 27 & 113 & 183 & 245 & 323 & 386 & 462 & & & & & & & & & & \\
\hline$\phi_{2}(\mathrm{a})$ & $\mathcal{E}$ & 0 & 3.1 & -2.7 & 3.2 & -2.5 & 3.2 & -1.7 & 3.1 & & & & & & & & & & \\
\hline
\end{tabular}



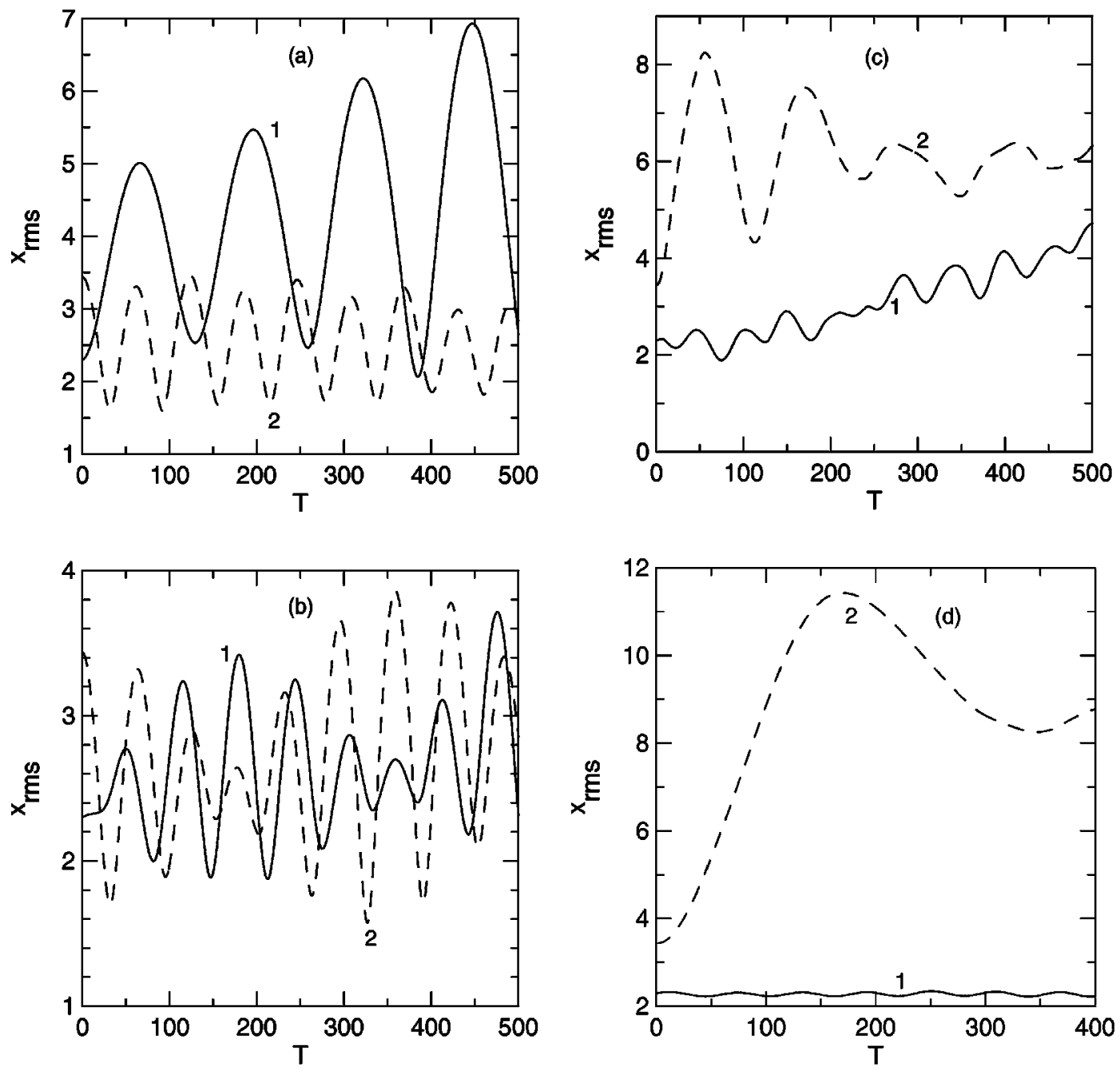

FIG. 5. The rms radii of the two components $\phi_{1}$ (full line) and $\phi_{2}$ (dashed line) of the wave function at different reduced times $T$ $\equiv t / 0.05$ for the oscillating condensate, when on the preformed condensate of model (a) of Fig. 1, we suddenly inflict the following changes: (a) $c_{1}=0.25$ and $c_{2}=1$; (b) $c_{1}=c_{2}=1$; (c) $n_{11}=n_{22}=5 ; n_{21}=n_{12}=100$; and (d) $c_{2}=0$ calculated with $\Delta=0.05$ and $\Delta_{1}=0.01$. All other parameters are maintained unchanged.

magnets responsible for confinement [16].

In the first type of problems we consider a sudden change of the harmonic-oscillator frequencies or scattering lengths at $t=0$ and study its effect on a preformed condensate. In the second type we study the effect of a periodic temporal variation of these frequencies on a preformed condensate. Finally, we study the effect of a periodic temporal variation of the scattering lengths on the preformed condensate. In all cases we take the preformed condensate as the one described by the model (a) of Fig. 1. We have commented before that the parameters of this model can simulate the coupled BEC composed of the ground and an excited state of ${ }^{87} \mathrm{Rb}$, which gives a motivation for this choice. When we implement these time-dependent perturbations, the system starts to oscillate (grow and shrink) with time. The corresponding evolution can be studied best through the rms radii [7], which execute periodic oscillation with time.

\section{A. Sudden change of trap frequency or scattering length}

By varying the external fields it is possible to vary the harmonic-oscillator trap frequency of the confining trap as well as the atomic interactions (scattering lengths) [17]. First we consider a sudden change of both the trap frequencies on the preformed coupled stationary BEC state corresponding to model (a) of Fig. 1. We set the reduced time $T \equiv t / 0.05=0$ when we start the time evolution. As the time step $\Delta$ is 0.05 , $T$ is just the number of iterations. In this model we have two different trap frequencies for the two components given by $c_{1}=1$ and $c_{2}=0.25$. In the first case at $T=0$ we suddenly interchange the constants $c_{1}$ and $c_{2}$, i.e., we set $c_{1}=0.25$ and $c_{2}=1$ and study the time evolution. The evolution of the rms radii corresponding to $\phi_{1}$ and $\phi_{2}$ are shown in Fig. 5(a). Both rms radii execute oscillations. However, that corresponding to $\phi_{1}$ has a much larger amplitude. The periods of oscillation of the two radii are different.

Next we consider a sudden change in one of the trap frequencies on the preformed condensate at $T=0$ corresponding to model (a) of Fig. 1 with $c_{1}=1$ and $c_{2}=0.25$. At $T=0$, we set $c_{1}=c_{2}=1$, which corresponds to parameters of model (c) of Fig. 1. The evolution of the rms radii is shown in Fig. 5(b). Although in the stationary configuration of 
model (c) of Fig. $1, \phi_{1}=\phi_{2}$, this condition is never attained in this evolution problem. The system keeps on oscillating indefinitely with time. The oscillation shown in Figs. 5(a) and 5(b) has nothing to do with the nonlinear or coupled nature of the problem. Similar oscillation also appears in an uncoupled linear oscillator when the trap frequency is suddenly changed. In the present coupled nonlinear problem both rms radii execute oscillations with time. However, when the amplitude of oscillation of one of the components increases, that of the other decreases. This behavior denotes the transfer of kinetic energy from one component to the other.

Now we study the effect of a sudden change of the scattering length(s) on the preformed condensate [17]. We consider the problem when the parameters of model (a) of Fig. 1 are suddenly changed to those of model (b) of Fig. 1 at $T$ $=0$. This is achieved by changing the nonlinearities suddenly at $T=0$ from $n_{11}=n_{22}=10, n_{12}=n_{21}=5$ to $n_{11}=n_{22}$ $=5, n_{12}=n_{21}=100$ with a variation of the external field that controls the scattering length(s). In this case the oscillations of the system are shown in Fig. 5(c), where we plot the time evolution of the rms radii of the two components. Both components of the condensate execute oscillations but with different frequencies and amplitudes. One of the components execute giant oscillations with large amplitude, whereas the amplitude of the other is much smaller.

Finally, we consider the case when one of the trapping potentials is switched off at $T=0$ on the preformed condensate of Fig. 1, model (a) by setting $c_{2}=0$. The oscillation in this case is shown in Fig. 5(d), where we plot the two rms radii. In the absence of the trapping potential the second component of the condensate can no longer remain localized in space. However, it does not expand monotonically before evaporating. It starts to execute giant oscillation and eventually escapes to infinity. Similar oscillation was found in the case of an uncoupled BEC when the trapping potential was removed [5]. The first component essentially remains unchanged during the process under the action of the unchanged trap potential. The minor oscillation of the rms radii of the first component is due to the coupling to the expanding second component.

\section{B. Periodic oscillation of trap frequency}

Instead of making a sudden change in the parameters of the model, next we introduce periodic oscillation in some of the parameters of the model for $T \geqslant 0$ and study the consequence on the system. We introduce a periodic variation in the parameters $c_{i}$, which are related to the harmonicoscillator trap frequencies. Experimentally, this variation is possible via a variation of the external fields, which are controlled by currents.

We again consider at $T=0$ the preformed condensate of the model (a) of Fig. 1. First we consider the variation $c_{1}$ $=1-0.5 \sin (\pi T / 20)$, which corresponds to varying the frequency of the first trap. The resultant variation of the two rms radii are shown in Fig. 6. The first radius (full line) oscillates more rapidly with larger amplitude and frequency than the second radius (dashed line). This is reasonable as we

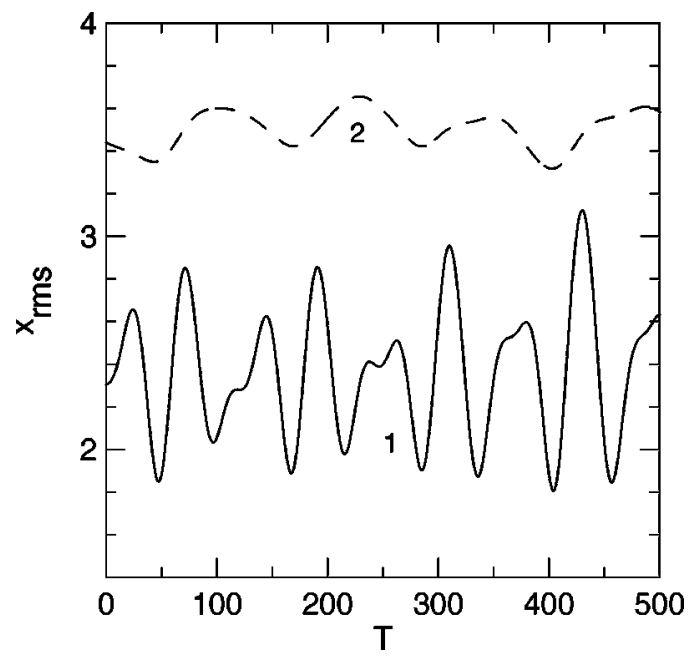

FIG. 6. The rms radii of the two components $\phi_{1}$ (full line) and $\phi_{2}$ (dashed line) of the wave function at different reduced times $T \equiv t / 0.05$ for the oscillating condensate, when on the preformed condensate of model (a) of Fig. 1, we suddenly inflict the following change: $c_{1}=1-0.5 \sin (\pi T / 20)$ calculated with $\Delta=0.05$ and $\Delta_{1}$ $=0.01$. All other parameters are maintained unchanged.

are directly varying the first frequency in this case. The rms radius of the second wave function feels the effect through its coupling to the first component. We also varied both the parameters $c_{1}$ and $c_{2}$ in a periodic fashion, which corresponds to varying both the frequencies. In this case both the rms radii execute oscillation. However, no interesting effect is observed and we do not show the details of that oscillation here.

\section{Periodic oscillation of scattering length}

Now we study the effect of a periodic variation of the scattering length(s) of the system on a preformed condensate. In our formulation this corresponds to a periodic variation of the parameters $n_{i l}$. This variation of the atomic interactions or the scattering lengths is now feasible experimentally [17]. We consider the periodic variation in one of the scattering lengths $\left(a_{11}\right)$ by setting $n_{11}=1-0.5 \sin (\pi T / 20)$ for $T \geqslant 0$ on the preformed condensate of model (a) of Fig. 1. The resultant oscillation of the rms radii are shown in Fig. 7. This variation corresponds to a variation of the atomic interaction among atomic states of the first type. Consequently, the rms radii of the first component of the BEC executes pronounced oscillation with moderate amplitude. There is no direct variation in the parameters of the second component. The second component of the condensate feels the effect of variation of $n_{11}$ through the coupling to the first component. Because of this secondary effect the second component also executes oscillation as can be seen from its rms radii in Fig. 7, albeit with a much smaller amplitude compared to the first component.

We also considered a periodic variation of the scattering length between one atom of each type $\left(a_{12}=a_{21}\right)$ by setting $n_{12}=n_{21}=0.5-0.25 \sin (\pi T / 20)$ on the same preformed condensate for $T \geqslant 0$ and studied the resultant oscillation of the 


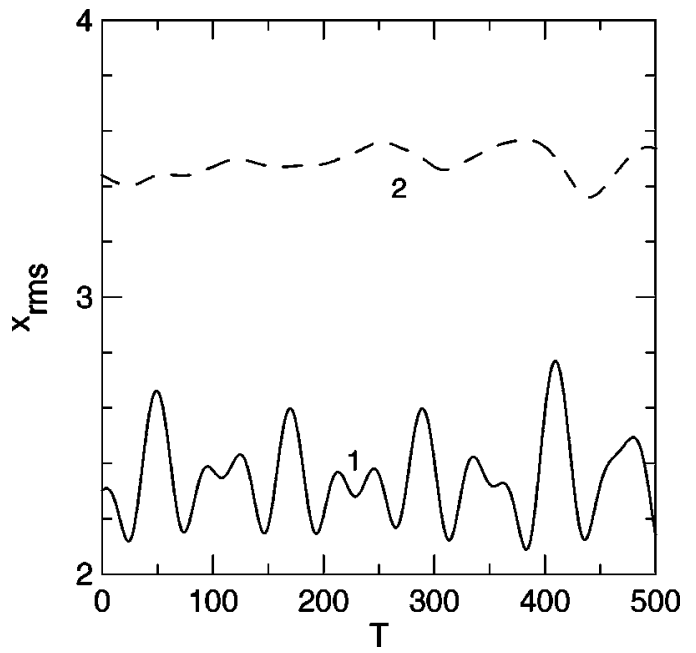

FIG. 7. The rms radii of the two components $\phi_{1}$ (full line) and $\phi_{2}$ (dashed line) of the wave function at different reduced times $T \equiv t / 0.05$ for the oscillating condensate, when on the preformed condensate of model (a) of Fig. 1, we suddenly inflict the following change: $n_{11}=1-0.5 \sin (\pi T / 20)$ calculated with $\Delta=0.05$ and $\Delta_{1}$ $=0.01$. All other parameters are maintained unchanged.

rms radii. However, no interesting behavior was observed and we do not show details of that oscillation.

\section{SUMMARY}

In this paper we present a numerical study of the coupled time-dependent Gross-Pitaevskii equation for BEC in three space dimensions under the action of harmonic-oscillator trap potentials with attractive and repulsive interparticle interactions between different types of atoms [13].

The time-dependent coupled GP equation is solved by discretizing it using a Crank-Nicholson-type scheme [5,15].
This method leads to good convergence for small nonlinearity. Numerical difficulty appears for large nonlinearity $\left(n_{i l}\right.$ $>20$ ). For medium nonlinearity, the accuracy of the method can be increased by reducing the space step $h$.

The ground-state stationary wave functions are found to be sharply peaked near the origin for attractive interatomic interaction for larger nonlinearity (Fig. 4). For a repulsive interatomic interaction the wave function extends over a larger region of space (Figs. 1 and 2). In the case of an attractive potential, the rms radii decrease with an increase of nonlinearity. There could be a collapse for attractive interaction when the nonlinear parameters $n_{i l}$ are increased as in the uncoupled case [8]. In the purely repulsive case we solved two and four coupled GP equations. In problems involving attraction we solved only the two coupled GP equations.

In addition to the stationary problem we studied three types of evolution problems. A stable coupled condensate is considered at $T=0$ on which a time-dependent perturbation is introduced. Two types of perturbations were considered on a two-component condensate with purely repulsive interactions. In the first type a sudden change in the parameters related to the frequencies of the trap and the scattering lengths was introduced. In the second type a periodic variation of the different scattering lengths and the frequencies of the harmonic-oscillator trap was introduced. In all cases the condensates execute periodic oscillation, which is studied via the time evolution of the rms radii as in the uncoupled case [7]. We conclude that the present time-dependent approach is very suitable for studying both the stationary and timeevolution problems of a coupled BEC.

\section{ACKNOWLEDGMENTS}

This work was supported in part by the CNPq and FAPESP of Brazil.
[1] M. H. Anderson, J. R. Ensher, M. R. Matthews, C. E. Wieman, and E. A. Cornell, Science 269, 198 (1995); K. B. Dadic, M. O. Mewes, M. R. Andrews, N. J. van Druten, D. S. Durfee, D. M. Kurn, and W. Ketterle, Phys. Rev. Lett. 75, 3969 (1995); D. G. Fried, T. C. Killian, L. Willmann, D. Landhuis, S. C. Moss, D. Kleppner, and T. J. Greytak, ibid. 81, 3811 (1998).

[2] P. A. Ruprecht, M. J. Holland, K. Burnett, and M. Edwards, Phys. Rev. A 51, 4704 (1995); Yu. Kagan, A. E. Muryshev, and G. V. Shlyapnikov, Phys. Rev. Lett. 81, 933 (1998).

[3] S. Giorgini, L. P. Pitaevskii, and S. Stringari, Phys. Rev. A 54, R4633 (1996); S. K. Adhikari and A. Gammal, Physica A 286, 299 (2000); M. J. Holland, D. S. Jin, M. L. Chiofalo, and J. Cooper, Phys. Rev. Lett. 78, 3801 (1997).

[4] A. Gammal, T. Frederico, and L. Tomio, Phys. Rev. E 60, 2421 (1999); A. Gammal, T. Frederico, L. Tomio, and F. Kh. Abdullaev, Phys. Lett. A 267, 305 (2000); S. K. Adhikari, ibid. 265, 91 (2000).

[5] S. K. Adhikari, Phys. Rev. E 62, 2937 (2000).

[6] M. L. Chiofalo, S. Succi, and M. P. Tosi, Phys. Lett. A 260, 86 (1999); M. M. Cerimele, M. L. Chiofalo, F. Pistella, S. Succi, and M. P. Tosi, Phys. Rev. E 62, 1382 (2000).

[7] F. Dalfovo, S. Giorgini, L. P. Pitaevskii, and S. Stringari, Rev. Mod. Phys. 71, 463 (1999).

[8] R. J. Dodd, M. Edwards, C. J. Williams, C. W. Clark, M. J. Holland, P. A. Ruprecht, and K. Burnett, Phys. Rev. A 54, 661 (1996); S. K. Adhikari, Physica A 284, 97 (2000); C. C. Bradley, C. A. Sackett, and R. G. Hulet, Phys. Rev. Lett. 78, 985 (1997); H. T. C. Stoof, Phys. Rev. A 49, 3824 (1994).

[9] E. P. Gross, Nuovo Cimento 20, 454 (1961); L. P. Pitaevskii, Zh. Éksp. Teor. Fiz. 40, 646 (1961) [Sov. Phys. JETP 13, 451 (1961)].

[10] M. R. Matthews, B. P. Anderson, P. C. Haljan, D. S. Hall, C. E. Wieman, and E. A. Cornell, Phys. Rev. Lett. 83, 2498 (1999).

[11] M. R. Matthews, D. S. Hall, D. S. Jin, J. R. Ensher, C. E. Wieman, E. A. Cornell, F. Dalfovo, C. Minniti, and S. Stringari, Phys. Rev. Lett. 81, 243 (1998).

[12] C. J. Myatt, E. A. Burt, R. W. Ghrist, E. A. Cornell, and C. E. Wieman, Phys. Rev. Lett. 78, 586 (1997).

[13] A. Sinatra, P. O. Fedichev, Y. Castin, J. Dalibard, and G. V. 
Shlyapnikov, Phys. Rev. Lett. 82, 251 (1999); S. Giovanazzi, A. Smerzi, and S. Fantoni, ibid. 84, 4521 (2000).

[14] B. D. Esry, C. H. Greene, J. P. Burke, Jr., and J. L. Bohn, Phys. Rev. Lett. 78, 3594 (1997); T.-L. Ho and V. B. Shenoy, ibid. 77, 3276 (1996).
[15] S. E. Koonin, Computational Physics (Benjamin/Cummings, Menlo Park, NJ, 1986), pp. 161-167.

[16] Y. Castin and R. Dum, Phys. Rev. Lett. 77, 5315 (1996).

[17] S. L. Cornish, N. R. Claussen, J. L. Roberts, E. A. Cornell, and C. E. Wieman, Phys. Rev. Lett. 85, 1795 (2000). 